\title{
Photophysical and Photochemical Properties and Aggregation Behavior of Phthalocyanine and Naphthalocyanine Derivatives
}

\author{
Thalita F. M. de Souza, Felipe C. T. Antonio, Mateus Zanotto, Paula Homem-de-Mello \\ and Anderson O. Ribeiro*
}

\author{
Centro de Ciências Naturais e Humanas, Universidade Federal do ABC (UFABC), \\ Avenida dos Estados, 5001, Bloco B, 09210-170 Santo André-SP, Brazil
}

\begin{abstract}
The photophysical and photochemical properties of phthalocyanine and naphthalocyanine with similar structures were studied in solution and with density-functional theory (DFT) computational method. The extended $\pi$-conjugated system in naphthalocyanines causes a bathochromic shift in UV-Vis, emission and excitation bands, and promotes lesser generation of singlet oxygen in solution when compared to phthalocyanines. Time dependent DFT (TD-DFT) calculations point out the molecular orbitals involved in Q-band transition, corresponding to highest occupied molecular orbital (HOMO) to lowest unoccupied molecular orbital (LUMO) transition with a concentration of charge along x-axis, while the transition to LUMO+1 is in y-axis direction. The presence of tert-butyl substituents does not affect the molecular orbitals shape, but affect their energies. Aggregation studies in dimethyl sulfoxide (DMSO):water solutions showed that naphthalocyanines studied have more aggregation tendency than the phthalocyanines. DFT studies indicated that stacked-dimers are preferred to rotated-stacked conformation due the interaction between $\mathrm{Zn}^{\text {II }}$ and nitrogen atom from different monomers.
\end{abstract}

Keywords: phthalocyanine, naphthalocyanine, aggregation, photophysical, computational studies

\section{Introduction}

Naphthalocyanines $(\mathrm{Nc})$ are phthalocyanines derivatives with extended $\pi$-conjugated system, which promotes a bathochromic shift in their absorption bands $(750-900 \mathrm{~nm}){ }^{1,2}$ Both macrocycles are widely applied as industrial dyes, in solar cells, ${ }^{3}$ nonlinear optics, ${ }^{4}$ electrochromic devices, ${ }^{5}$ photodynamic therapy (PDT) ${ }^{6}$ and chemical sensors. ${ }^{7}$

Phthalocyanines $(\mathrm{Pc})$ and naphtalocyanines have a high tendency to aggregate, especially in aqueous solution. This aggregation influences their photophysical and photochemical properties leading to disadvantages such as fluorescence quenching, lesser generation of singlet oxygen and loss of catalytic activity. ${ }^{8,9}$

Several works demonstrated that the aggregation behavior is influenced by many factors, e.g. strong $\pi-\pi$ electron interactions, coulombic forces, central metal, position and size of the substituents, axial substituents on the central metal, temperature and solvent. ${ }^{10,11}$ However,

\footnotetext{
*e-mail: anderson.ribeiro@ufabc.edu.br
}

detailed studies comparing Pc and Nc aggregation in solution are lacking. ${ }^{12,13}$

In the current study, we investigate the impact of the extended $\pi$-conjugation in macrocycles with same peripheral substituents (Figure 1). We evaluated the photophysical and photochemical properties in DMSO solution, and their aggregation in DMSO:water mixtures.

\section{Experimental}

Materials

All reagents and solvents were used as received. Zinc phthalocyanine ( $\mathrm{ZnPc})$ and zinc-tert-butylphthalocyanine (1) were purchased from Sigma-Aldrich. The zinc-naphthalocyanine $(\mathrm{ZnNc})^{14}$ and zinc-tert-butylnaphthalocyanine (2) were synthesized ${ }^{15,16}$ as described.

\section{Equipment}

Fourier transform infrared (FTIR) spectra were obtained on a Varian FTIR-660 spectrophotometer with the sample 


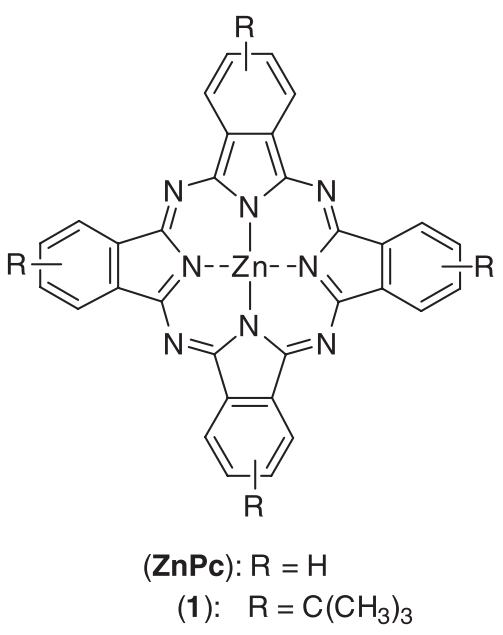

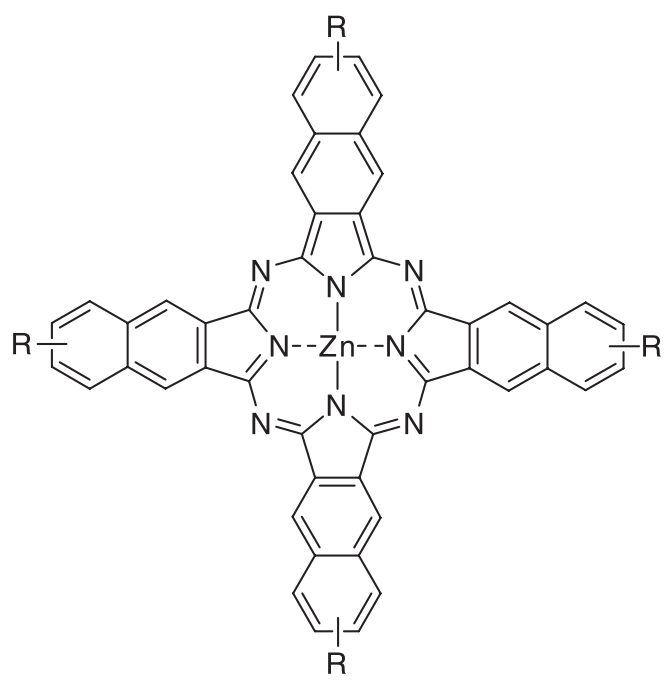

$(\mathrm{ZnPc}): \mathrm{R}=\mathrm{H}$

(2): $\quad \mathrm{R}=\mathrm{C}\left(\mathrm{CH}_{3}\right)_{3}$

Figure 1. Structure of phthalocyanines and naphthalocyanines investigated in this study.

prepared as $\mathrm{KBr}$ pellet. ${ }^{1} \mathrm{H}$ nuclear magnetic resonance (NMR) spectra were performed with $\mathrm{CDCl}_{3}$ or DMSO- $d_{6}$ as solvent and tetramethylsilane (TMS) as internal standard.

All absorption spectra were taken on a Varian Cary $50 \mathrm{UV}$-Vis spectrometer and fluorescence emission and excitation spectra using a Varian Eclipse fluorescence spectrophotometer.

Fluorescence decay profiles and singlet oxygen quantum yield were measured using a time-correlated single photon count (TCSPC) spectrometer (Fluotime 300, PicoQuant) with a laser diode emitting $634 \mathrm{~nm}$ (LDH-D-C-634, PicoQuant). The data was analyzed with the program Fluofit, PicoQuant. All measurements were performed at room temperature.

\section{Synthesis}

Scheme 1 presents the experimental conditions and the compounds synthesized to obtain the naphthalocyanines applied in this study.

\section{General procedure for precursor synthesis}

To a solution of $4 \mathrm{mmol}$ of $o$-xylene or tert-butyl$o$-xylene in $12.0 \mathrm{~mL}$ of dry $\mathrm{CH}_{2} \mathrm{Cl}_{2}, 14 \mathrm{mmol}$ of $\mathrm{N}$-bromosuccinimide were added. The reaction mixture was refluxed and irradiated with a $125 \mathrm{~W}$ mercury vapor lamp for $6 \mathrm{~h}$. The mixture was filtered while hot and the filtrate was evaporated to obtain a yellowish solid. The crude product was dissolved in $12.0 \mathrm{~mL}$ of dry dimethylformamide (DMF), and $4 \mathrm{mmol}$ of fumaronitrile and $20 \mathrm{mmol}$ of dry NaI were added to the solution. The stirred solution was kept for $12 \mathrm{~h}$ at $70-80{ }^{\circ} \mathrm{C}$. The dark reaction mixture was added dropwise to a stirred solution of $20 \mathrm{mmol}$ of sodium thiosulfate in $300 \mathrm{~mL}$ of water. The yellow precipitate was filtered off, washed with water and acetone, and then purified by a silica-gel column with hexane:ethyl acetate $(2: 1)$ as eluent.

\section{2,3-Dicyanonaphthalene (3)}

Yield 57\%; IR (KBr pellet) $\mathrm{v} / \mathrm{cm}^{-1}$ 3097-3031 (Ar-H), 2971-2867 (aliph. C-H), 2227 (C $\equiv \mathrm{N}), 1716,1618,1361$, 1274, 1082; ${ }^{1} \mathrm{H}$ NMR (400 MHz, $\left.\mathrm{CDCl}_{3}\right) \delta 8.37$ (s, 2H), $8.00(\mathrm{~m}, 2 \mathrm{H}), 7.81(\mathrm{~m}, 2 \mathrm{H})$.

\section{6-tert-Butyl-2,3-dicyanonaphthalene (4)}

Yield 33\%; IR (KBr pellet) $\mathrm{v} / \mathrm{cm}^{-1}$ 3099-3000 (Ar-H), 2990-2800 (aliph. C-H), 2229 (C $\equiv \mathrm{N}), 1703,1622,1363$, 1261, 1097; ${ }^{1} \mathrm{H}$ NMR (400 MHz, $\mathrm{CDCl}_{3}$ ) $\delta$ 8.32-8.31 (d, J 6.82 Hz, 2H), 7.94-7.87 (m, 3H), 1.44 (s, 9H).

General procedure for naphthalocyanines synthesis

$0.43 \mathrm{mmol}$ of $\mathbf{3}$ or $\mathbf{4}$ and $0.21 \mathrm{mmol}$ of zinc(II) acetate were suspended in $1 \mathrm{~mL}$ dry pentanol at $150^{\circ} \mathrm{C}$, under inert gas. Then, $0.43 \mathrm{mmol}$ of 1,8-diazabicyclo[5.4.0]undec7 -ene (DBU) was added and the solution was stirred for $12 \mathrm{~h}$ at $150{ }^{\circ} \mathrm{C}$. After cooling, methanol was added and the resulted precipitate was centrifuged.

\section{Zinc-naphthalocyanine ( $\mathrm{ZnNc})$}

The crude product was purified by a silica-gel column with tetrahydrofuran (THF)/DMF (7:3) as eluent. Yield 15\%; IR (KBr pellet) $v / \mathrm{cm}^{-1} 3100-3000(\mathrm{Ar}-\mathrm{H})$, 
<smiles>[R]c1ccc(C)c(C)c1</smiles><smiles>[R]c1ccc2cc(C#N)c(C#N)cc2c1</smiles>

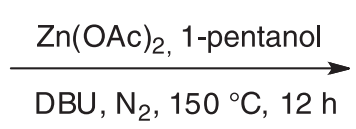

(3) $\mathrm{R}=\mathrm{H}$

(4) $\mathrm{R}=\mathrm{C}\left(\mathrm{CH}_{3}\right)_{3}$

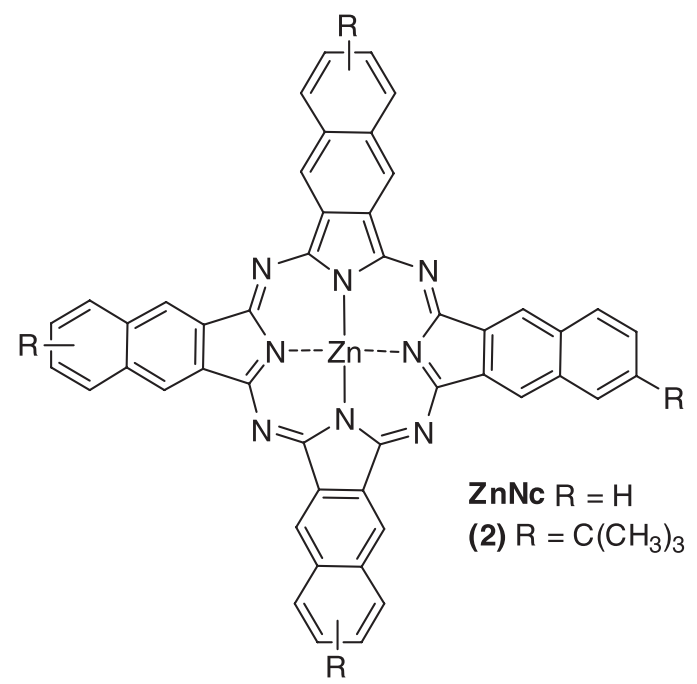

Scheme 1. Synthesis of zinc-naphthalocyanine $(\mathrm{ZnNc})$ and zinc-tert-butyl-naphthalocyanine (2).

2990-2800 (aliph. C-H), 1716, 1653, 1433, 1354, 1149, 1088; ${ }^{1} \mathrm{H}$ NMR (400 MHz, THF- $\left.d_{4}\right) \delta 8.49-8.40(\mathrm{~m}, 8 \mathrm{H})$, 8.11-8.02 (m, 8H), 7.90-7.80 (m, 8H).

\section{Zinc-tert-butyl-naphthalocyanine (2)}

The crude product was purified by a silica-gel column with toluene/THF (10-30\%) as eluent. Yield 18\%; IR ( $\mathrm{KBr}$ pellet) $v / \mathrm{cm}^{-1}$ 3090-3000 (Ar-H), 2990-2820 (aliph. C-H), $1714,1460,1357,1101 ;{ }^{1} \mathrm{H}$ NMR (400 MHz, DMSO- $d_{6}$ ) $\delta$ 8.43-8.40 (d, $J 9.14 \mathrm{~Hz}, 8 \mathrm{H}), 8.21-8.18(\mathrm{~d}, J 9.60 \mathrm{~Hz}$, $8 \mathrm{H}), 7.91-7.89(\mathrm{~d}, J 1.83 \mathrm{~Hz}, 2 \mathrm{H}), 7.88-7.87(\mathrm{~d}, J 2.06 \mathrm{~Hz}$, $2 \mathrm{H}), 1.41$ (s, 36H).

\section{Fluorescence quantum yields and lifetimes}

Fluorescence quantum yields $\left(\Phi_{\mathrm{F}}\right)$ were determined in DMSO by the comparative method using equation $1 . .^{17,18}$

$\Phi_{\mathrm{F}}=\Phi_{\mathrm{F}}^{\mathrm{Std}} \frac{\mathrm{F} \times \mathrm{A}_{\mathrm{Std}} \times \mathrm{n}^{2}}{\mathrm{~F}_{\mathrm{Std}} \times \mathrm{A} \times \mathrm{n}_{\mathrm{Std}}^{2}}$

where $\mathrm{F}$ and $\mathrm{F}_{\mathrm{Std}}$ are the areas under the fluorescence emission curves of the samples and the standard, respectively; $\mathrm{A}$ and $\mathrm{A}_{\mathrm{Std}}$ are the relative absorbance of the samples and standard at the excitation wavelength, respectively; $\mathrm{n}^{2}$ and $\mathrm{n}_{\mathrm{Std}}^{2}$ are the refractive indices of solvents for the sample and standard, respectively. Unsubstituted $\mathrm{ZnPc}$ (in DMSO) $\left(\Phi_{\mathrm{F}}=0.20\right)^{17,18}$ was employed as the standard. Both the sample and standard were excited at $350 \mathrm{~nm}$. The absorbance of the solutions ranged between 0.06 and 0.09 at the excitation wavelength.

The fluorescence lifetime $\left(\tau_{\mathrm{F}}\right)$ values were directly determined in DMSO by TCSPC method. Both the sample and standard were excited at $634 \mathrm{~nm}$.

Singlet oxygen quantum yields

Singlet oxygen quantum yield $\left(\Phi_{\Delta}\right)$ values were determined in air by direct detection of the singlet oxygen phosphorescence at $1270 \mathrm{~nm}$. The $\Phi_{\Delta}$ were determined in THF by the comparative method using equation $2 .{ }^{19}$

$\Phi_{\Delta}=\Phi_{\Delta}^{\mathrm{Std}} \frac{\mathrm{I} \times \mathrm{A}_{\mathrm{Std}}}{\mathrm{I}_{\mathrm{Std}} \times \mathrm{A}}$

where $I$ and $I_{S t d}$ are the phosphorescence intensity of singlet oxygen at $1270 \mathrm{~nm}$ of the samples and the standard, respectively. Unsubstituted $\mathrm{ZnPc}$ (in THF) $\left(\Phi_{\Delta}=0.53\right)^{20}$ 
was employed as the standard. Both the sample and standard were excited at $634 \mathrm{~nm}$. The concentration of the compounds was $5 \mu \mathrm{M}$.

\section{Computational studies}

Density-functional theory (DFT) approach was employed to obtain the geometry of the compounds in a minimum in the potential energy surface by using the B3LYP functional and the 6-311G(d,p) basis set. Time dependent DFT (TD-DFT) was performed for 80 states in order to obtain the electronic spectra of the compounds by using the same methodology. Intramolecular vibrations were not included since they are negligible for rigid molecules as those studied here. ${ }^{21}$ Solvent effects were included by means of the IEFPCM continuum model. ${ }^{22}$ These calculations were carried out with Gaussian09 package. ${ }^{23}$

In order to give insight on how absorption spectrum at low concentrations of phthalocyanine is affected by the presence of aggregates, we performed DFT calculations by means of BLYP functional and 6-311G(d) basis set with Grimme's correction for dispersion interactions (D3-BJ) ${ }^{24,25}$ as implemented in ORCA software ${ }^{26}$ for dimers of $\mathrm{ZnPc}$ and $\mathrm{ZnNc}$. We started geometry optimization with different stacking conformations, by changing the rotation angle of one monomer in relation to the other. This methodology was evaluated for free phthalocyanine previously. ${ }^{27}$ Absorption spectra were obtained for the different dimer conformations found using the same methodology employed for monomer calculations.

\section{Results and Discussion}

\section{Synthesis and characterization}

The synthesis of naphthalonitriles was realized by a multistep procedure ${ }^{14-16}$ starting from $o$-xylene or tert-butyl$o$-xylene by bromation with $N$-bromosuccinimide (NBS) following by a cyclisation with fumarodinitrile. Naphthalocyanines were synthesized in 1-pentanol, at $150{ }^{\circ} \mathrm{C}$, using DBU as catalyst, under $\mathrm{N}_{2}$ atmosphere. All compounds were characterized by spectroscopic methods including UV-Vis, IR and ${ }^{1} \mathrm{H}$ NMR. The results are consistent with the proposed structures.

In the IR absorption spectra, the formation of phthalocyanine was confirmed by disappearance of phthalonitrile $\mathrm{C} \equiv \mathrm{N}$ band at $2226 \mathrm{~cm}^{-1}$. The ${ }^{1} \mathrm{H}$ NMR spectra of 2 exhibited aromatic ring protons with $\delta$ ranging from 7.88 to $8.43 \mathrm{ppm}$ and aliphatic protons at $1.41 \mathrm{ppm}$ (Figure 2).

The UV-Vis spectra of macrocyclic compounds were recorded in DMSO (Figure 3). The complexes exhibited a very intense $\mathrm{Q}(0,0)$ band in the $600-800 \mathrm{~nm}$ region and a Soret band between $300-400 \mathrm{~nm}$. Table 1 summarizes the wavelengths and associated extinction coefficients in Q-band. Due to the extended conjugation, the Q-band maximum was red-shifted around $100 \mathrm{~nm}$ for $\mathbf{2}$ and $\mathrm{ZnNc}$ compared to the phthalocyanine band.

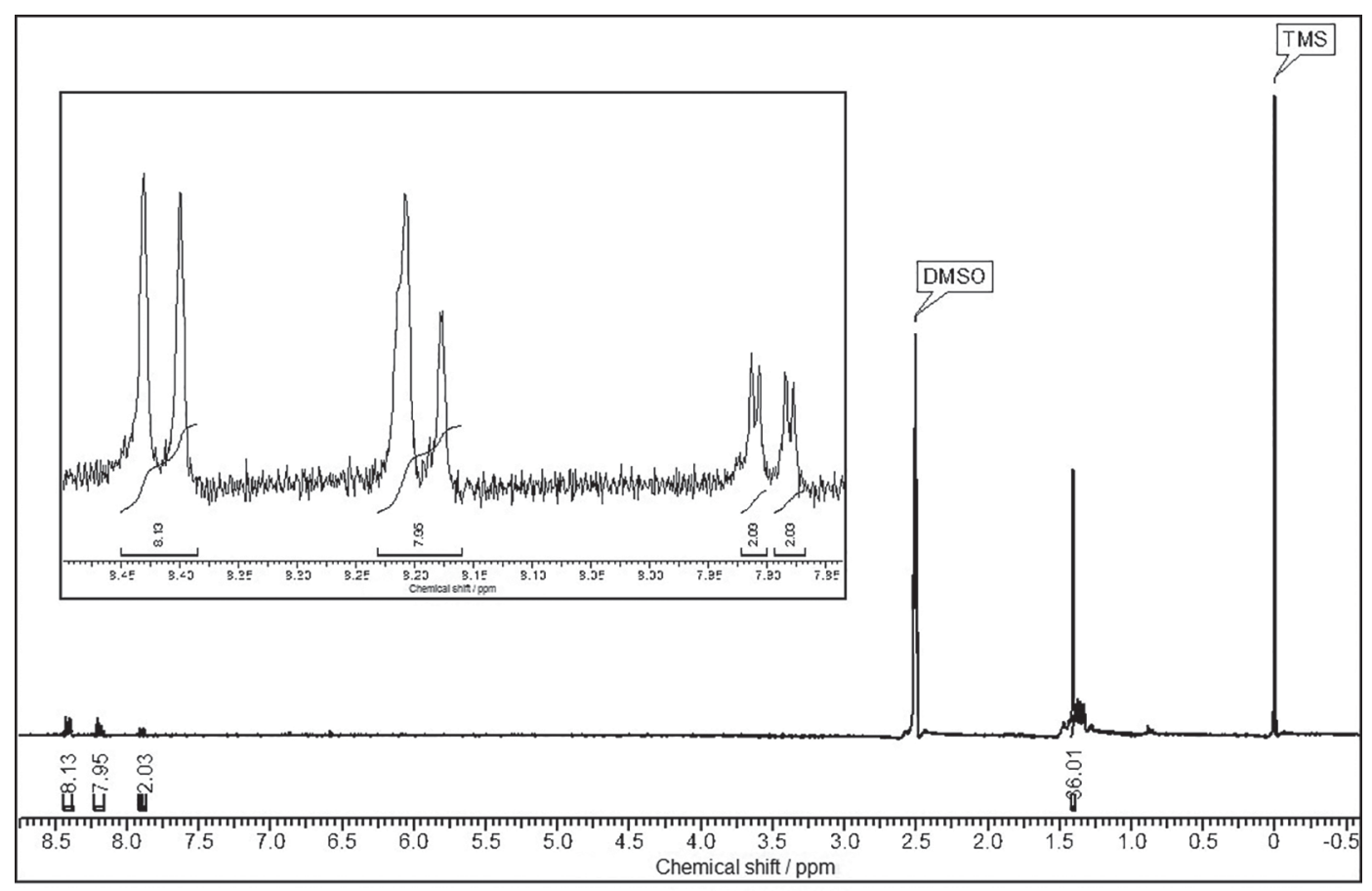

Figure 2. ${ }^{1} \mathrm{H}$ NMR spectrum (500 MHz, DMSO- $d_{6}$ ) of compound 2. 


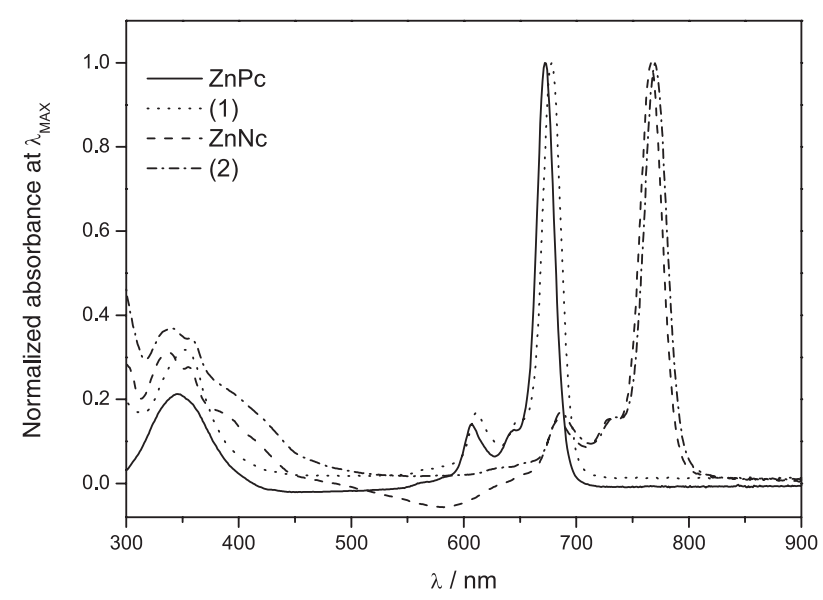

Figure 3. Normalized absorption spectra of $\mathrm{ZnPc}, \mathbf{1}, \mathrm{ZnNc}$ and $\mathbf{2}$ in DMSO.

TD-DFT calculations point out the molecular orbitals involved in Q-band transition. This band is composed by two transitions: $\mathrm{HOMO} \rightarrow \mathrm{LUMO}$ and $\mathrm{HOMO} \rightarrow \mathrm{LUMO}+1$ (HOMO: highest occupied molecular orbital; LUMO: lowest unoccupied molecular orbital). Figure 4 presents the molecular orbitals for $\mathrm{ZnPc}$ and $\mathrm{ZnNc}$ which correspond to the already known behavior: HOMO to LUMO transition consists in a concentration of charge along $\mathrm{x}$-axis, while the transition to LUMO+1 is in y-axis direction. The same occurs for $\mathrm{ZnNc}$, but as the conjugated system is larger, the concentration of charge is more extended along each axis. Substitutions with tert-butyl groups do not affect expressively this behavior (molecular orbitals for $\mathbf{1}$ and $\mathbf{2}$ are presented in Figure S1, Supplementary Information (SI) section), and affect slightly molecular orbitals energies (Table 1).

In region between 300 and $400 \mathrm{~nm}$, there are degenerated transitions from HOMO-1 to LUMO/LUMO+1. For naphthalocyanines, besides those transitions, there are also transitions from HOMO to higher LUMOs, and for compound $\mathbf{2}$, those transitions are not degenerated due to tert-butyl substitutions (Table S1, SI section).

These molecular orbitals are in good agreement with previous simulations (see Ueno $\mathrm{et} \mathrm{al}^{28}$ for example), but it is interesting to note that HOMO- 1 for $\mathrm{ZnPc}$ does not include the nitrogen in meso positions ( $N$-meso), while metal-free phthalocyanine does, those nitrogen atoms participation
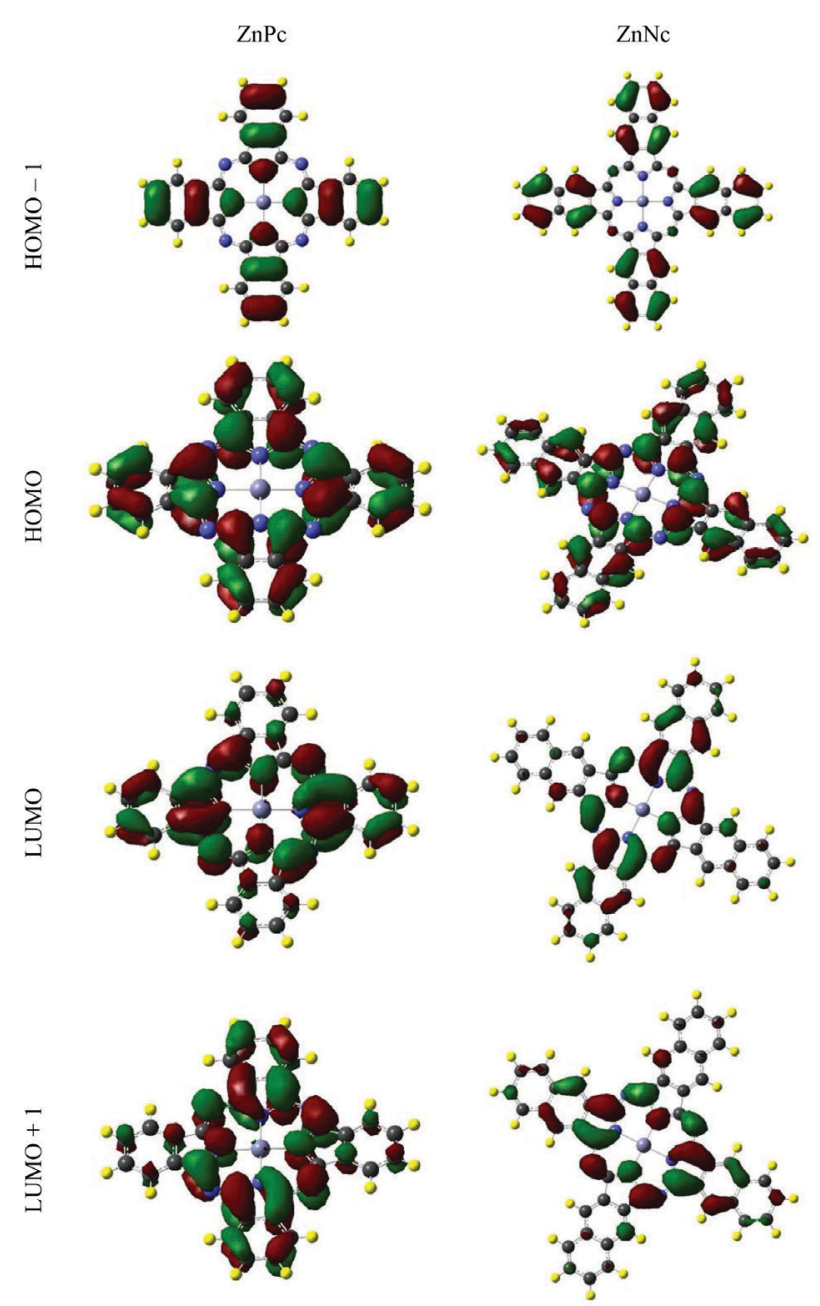

Figure 4. Molecular orbitals (HOMO-1, HOMO, LUMO and LUMO+1) involved in transitions that occur in Q-band region for $\mathrm{ZnPc}$ and $\mathrm{ZnNc}$. Hydrogen atoms are represented by yellow spheres, nitrogen in blue, carbon in dark grey and zinc in light grey (molecular orbitals for substituted compounds are presented in Supplementary Information section).

on HOMO-1 causes a well-known stabilization of the energy of the level. This phenomena is also observed for other metallophthalocyanines (particularly for NiPc; for some other metallic cations, HOMO-1 correspond to 3d-like orbitals). ${ }^{29,30}$ For naphthalocyanines, $N$-meso atoms participate in HOMO-1, but its contribution is small.

For all compounds, a small band appears next to Q-band. In TD-DFT calculated spectra (data presented in

Table 1. Experimental absorption, excitation and emission data for compounds in DMSO and TD-DFT molecular orbital energies

\begin{tabular}{lccccccc}
\hline Compound & Q-band $\lambda_{\max }^{\text {Abs }} / \mathrm{nm}$ & $\log \varepsilon$ & $\mathrm{E}_{\text {номо }} /$ a.u. & $\mathrm{E}_{\text {Luмо }} /$ a.u. & gap номо-Luмо $/$ a.u. & Excitation $\lambda_{\max }^{\text {exc }} / \mathrm{nm}$ & Emission $\lambda_{\max }^{\text {ems }} / \mathrm{nm}$ \\
\hline $\mathrm{ZnPc}$ & 675 & 5.2 & -0.1897 & -0.1096 & 0.0801 & 607 & 681 \\
$\mathbf{1}$ & 678 & 4.5 & -0.1825 & -0.1036 & 0.0789 & 612 & 686 \\
$\mathrm{ZnNc}$ & 766 & 5.0 & -0.1746 & -0.1058 & 0.0688 & 686 & 778 \\
$\mathbf{2}$ & 770 & 5.1 & -0.1700 & -0.1017 & 0.0683 & 688 & 783 \\
\hline
\end{tabular}


Supplementary Information), there are no transitions in this region, so those bands may not be due to purely electronic transition of monomeric species.

\section{Fluorescence spectra, quantum yields and lifetimes}

Fluorescence measurements of the compounds were recorded in DMSO (Table 1). The excitation spectra were similar to absorption spectra and both were mirror images of the emission spectra (Figure 5). The proximity of the wavelength of Q-band maxima of the absorption and excitation spectra for all compounds suggests that the nuclear configurations of the ground and excited states are similar and not affected by excitation. ${ }^{10}$ Due to the extended conjugation, the Q-band maxima of the excitation spectra and emission was red-shifted in the same order of absorption spectra.

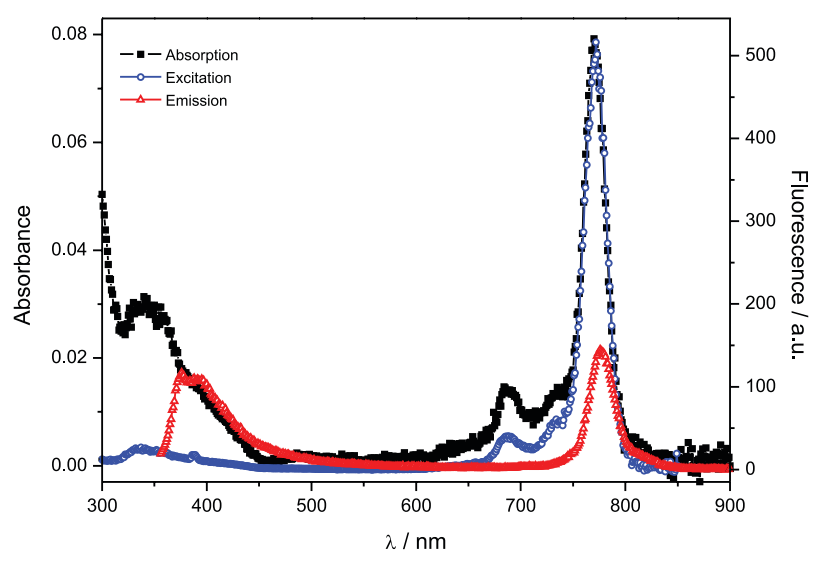

Figure 5. Absorption, excitation and emission spectra of $\mathrm{ZnNc}$ in DMSO. Concentration $=1.0 \times 10^{-6} \mathrm{~mol} \mathrm{~L}^{-1}$. Excitation wavelength $=350 \mathrm{~nm}$.

The fluorescence quantum yields $\left(\Phi_{\mathrm{F}}\right)$ of the compounds were determined by comparative method (Table 2). The values for phthalocyanines were higher than for naphthalocyanine. The fluorescence lifetime $\left(\tau_{\mathrm{F}}\right)$ of the compounds were determined by using TCSPC and, again, the values were lower for naphthalocyanines than phthalocyanines. These results suggest that the addition of benzene rings to the periphery of phthalocyanine macrocycle increases the non-radiative losses from excited states and decreases the efficiency of fluorescence process.

\section{Singlet oxygen quantum yields $\left(\Phi_{\Delta}\right)$}

The quantum yield of singlet oxygen generation was determined using the equation 2 by directed method measuring phosphorescence intensity of singlet oxygen. The $\Phi_{\Delta}$ values of $\mathrm{ZnPc}$ are higher than for other compounds (Table 2). Once more, higher extended $\pi$-conjugation in
Table 2. Photophysical data of compounds in DMSO

\begin{tabular}{lccc}
\hline Compound & $\Phi_{\mathrm{F}}$ & $\tau_{\mathrm{F}} / \mathrm{ns}$ & $\Phi_{\Delta}{ }^{\mathrm{a}}$ \\
\hline $\mathrm{ZnPc}$ & 0.20 & 3.3 & 0.53 \\
$\mathbf{1}$ & 0.18 & 3.2 & 0.34 \\
$\mathrm{ZnNc}$ & 0.07 & 2.5 & 0.41 \\
$\mathbf{2}$ & 0.08 & 2.4 & 0.29 \\
\hline
\end{tabular}

In THF,

naphthalocyanine results in lower values for singlet oxygen generation.

\section{Aggregation studies}

The UV-Vis spectra of compounds were obtained in DMSO, DMF, toluene, dichloromethane (DCM) and chloroform, both experimentally (Figure 6 for 2 ) and computationally (see Table S2, SI section). These results are in good agreement and show that the solvent influence in the absorption spectra is related only to small changes in bands intensity, due to the different dielectric constants and van der Waals radii (both affect the transition dipole moment and, consequently, the oscillator strength). The compounds showed monomeric behavior in these solvents, in the concentration range studied (from $1 \times 10^{-6}$ to $1 \times 10^{-5} \mathrm{~mol} \mathrm{~L}^{-1}$ ), as it is possible to see in DMSO in Figure $7 b$.

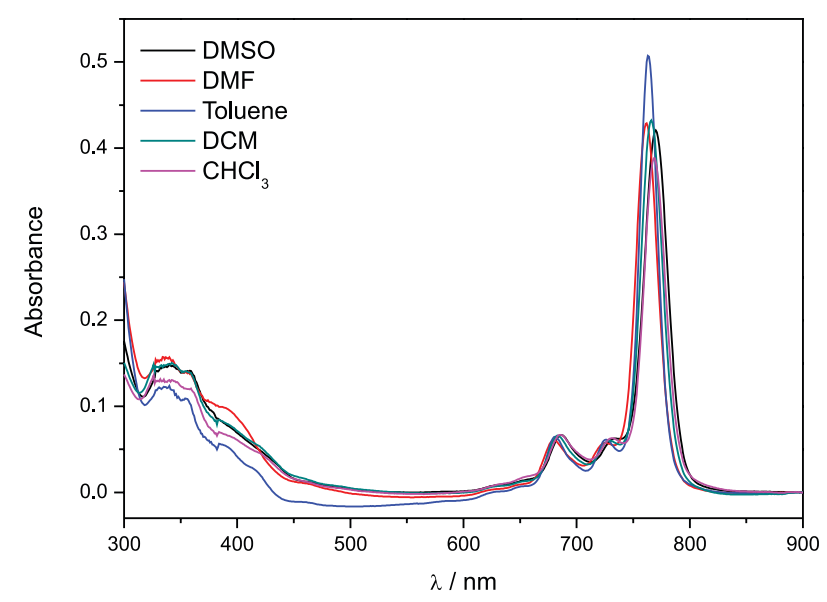

Figure 6. Absorption spectra of 2 in different solvents at $5.0 \times 10^{-6} \mathrm{~mol} \mathrm{~L}^{-1}$.

The aggregation was investigated in DMSO:water solutions. The absorption spectra of $\mathbf{2}$ in solution of DMSO:water for different water volume percentage are shown in Figure 8. The increase of the amounts of water in the DMSO:water mixtures leads to a decrease in the Q-band maximum intensity, demonstrating the formation of aggregated species. By plotting the intensity 

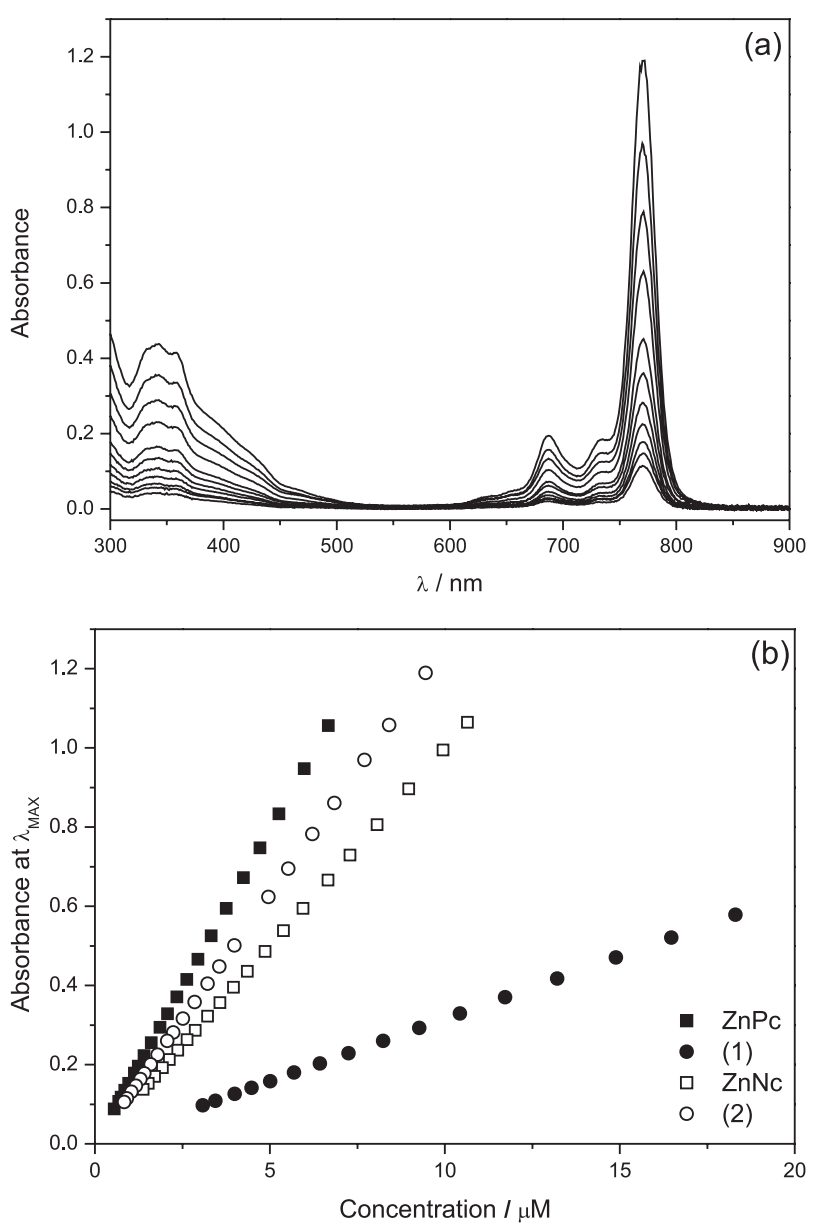

Figure 7. (a) Absorption spectra of 2 in DMSO from $8.4 \times 10^{-7}$ to $9.4 \times 10^{-6} \mathrm{~mol} \mathrm{~L}^{-1}$; (b) intensity at maximum absorption wavelength versus concentration for all studied compounds, in DMSO.

at maximum absorption wavelength versus water content, a bleaching of $50 \%$ in absorbance was observed at 35,30 , 20 and $15 \%$ of water for $\mathrm{ZnPc}, \mathbf{1}, \mathrm{ZnNc}$ and $\mathbf{2}$, respectively (Figure $8 \mathrm{~b}$ ). The Q-band has a suddenly decrease in their maximum intensity when the water volume percentage was higher than 60, 40, 30 and 40\% for $\mathrm{ZnPc}, \mathbf{1}, \mathrm{ZnNc}$ and $\mathbf{2}$, respectively (Figure 8a).

The fluorescence spectra of $\mathbf{2}$ in solution of DMSO:water for different water volume percentage are shown in Figure 9. The increase of the amounts of water in the DMSO:water mixtures causes a bathochromic shift in fluorescence emission spectra for all compounds. In addition, we observed a decrease in the fluorescence intensity, probably due to the aggregation of macrocycles. By plotting the fluorescence intensity at maximum emission wavelength versus water content, a bleaching of $50 \%$ in fluorescence intensity was observed at $35,35,20$ and $15 \%$ of water for $\mathrm{ZnPc}, \mathbf{1}, \mathrm{ZnNc}$ and 2, respectively. The results were similar to that obtained in absorbance experiment.

In order to quantify the impact of the $\pi$-conjugated system interaction on absorption spectra, we performed
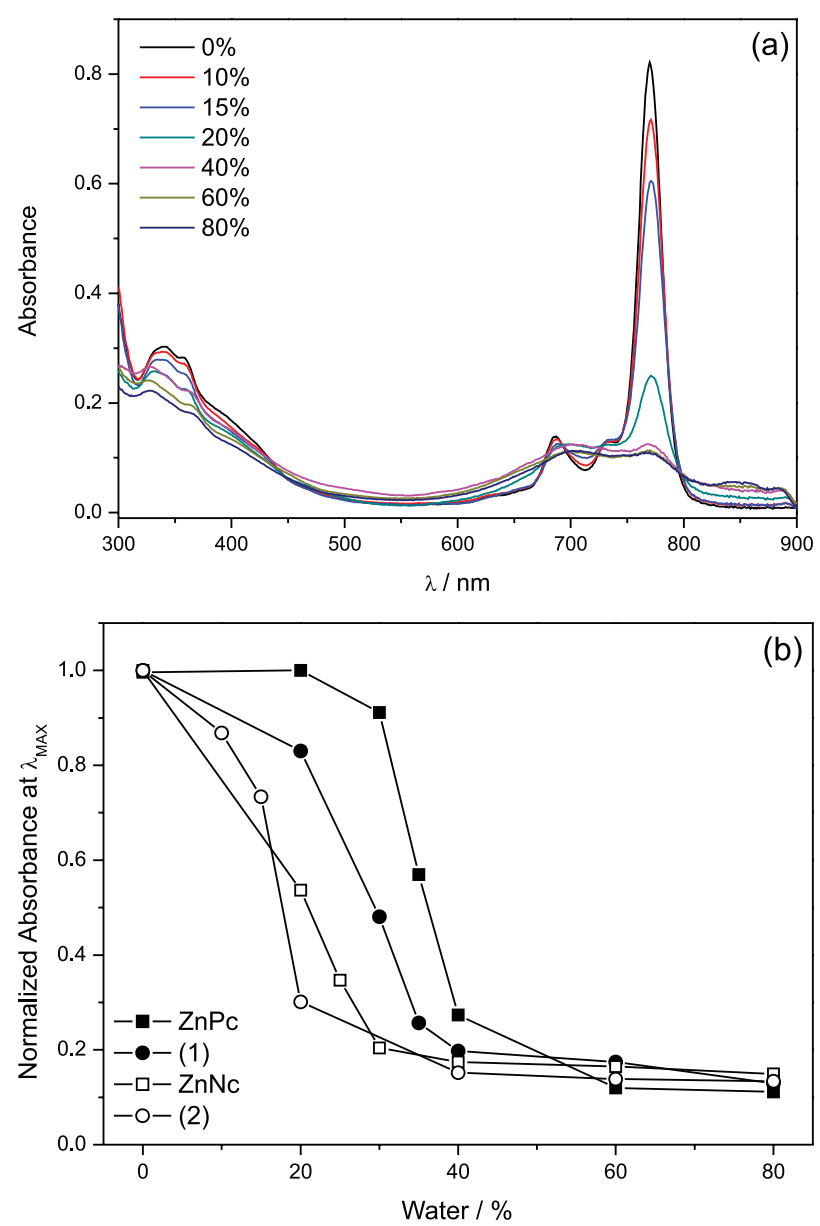

Figure 8. (a) Absorption spectra of $\mathbf{2}$ in DMSO/water solutions, water volume percentage as a parameter; (b) intensity at maximum absorption wavelength versus water percentage for all studied compounds.

DFT calculations with dispersion corrections for $\mathrm{ZnPc}$ and $\mathrm{ZnNc}$ dimers. Table 3 presents the energy for different conformations of dimers. The geometry optimization conducted for both compounds, $\mathrm{ZnPc}$ and $\mathrm{ZnNc}$, resulted in two conformations. In the first one, monomers are in the same orientation for both compounds $\left(0^{\circ}\right.$ of rotation), slightly displaced in the xy plane by $2 \AA$, and at $2.7 \AA$ distance between monomers, in good agreement with previous work ${ }^{31}$ and the interaction energy for $\mathrm{ZnNc}$ is even larger.

In the second conformation, monomers are superimposed, but rotated about $45^{\circ}$ and the distance between monomers for $\mathrm{ZnNc}$ is $0.7 \AA$ larger than for $\mathrm{ZnPc}$, what results in a smaller interaction. Structures with $0^{\circ}$ of rotation are probably the most stable because the relative position of each monomer allows the zinc atom to interact with nitrogen of the other monomer (Figure S3, SI section).

Moreover, in crystals of phthalocyanines and naphthalocyanines, there are only $0^{\circ}$ of rotation aggregates, and the structures that allow the interaction between $\mathrm{Zn}$ and $N$-meso are known as $\beta$-polymorphs. Electrostatic 

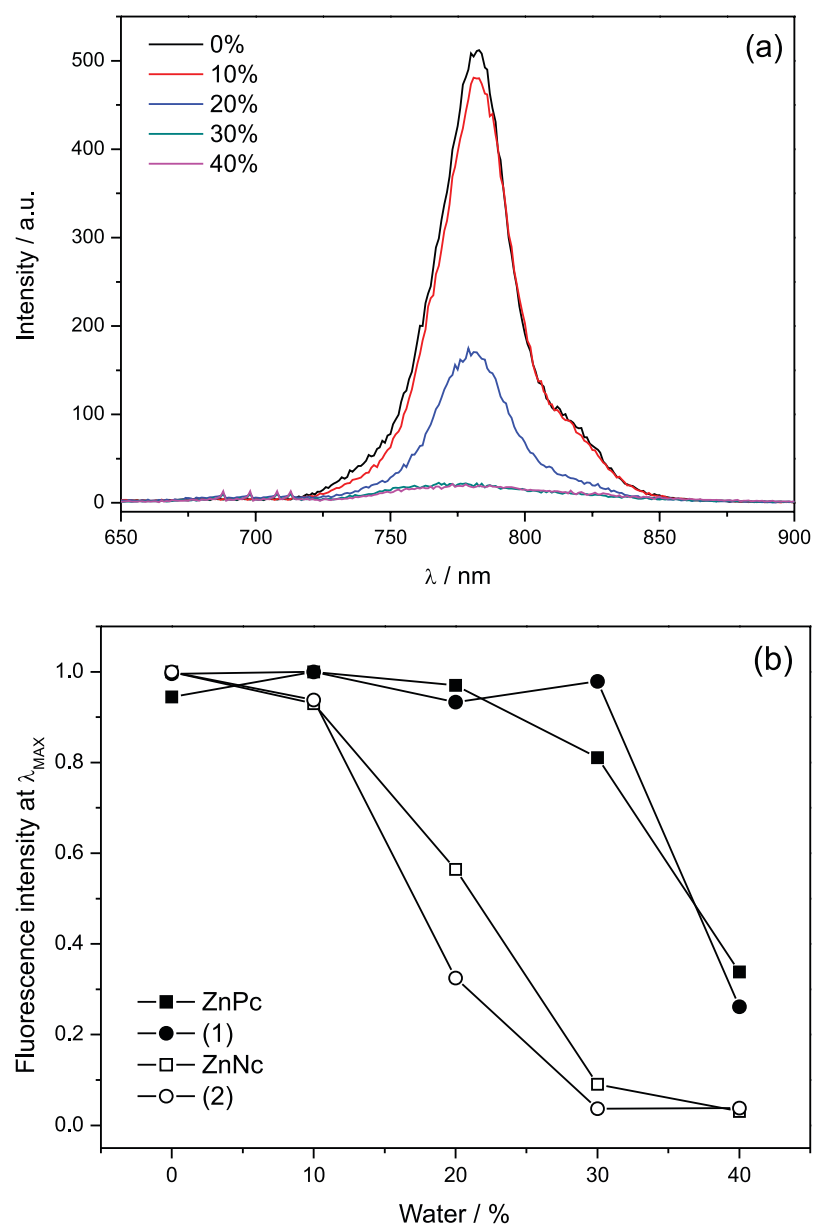

Figure 9. (a) Emission spectra of 2 in DMSO/water solution, water volume percentage as a parameter; (b) fluorescence intensity at maximum emission wavelength versus water percentage for all studied compounds.

Table 3. Aggregation energy $\left(\mathrm{E}_{\mathrm{dim}}\right)$ and structural parameters for dimers found for $\mathrm{ZnPc}$ and $\mathrm{ZnNc}$ : approximate rotation angle of one monomer in relation to the other and distance between monomers

\begin{tabular}{lccc}
\hline Compound & $\begin{array}{c}\text { Rotation angle / } \\
\text { degree }\end{array}$ & Distance / $\AA$ & $\begin{array}{c}\mathrm{E}_{\text {dim }} / \\
\left(\mathrm{kcal} \mathrm{mol}^{-1}\right)\end{array}$ \\
\hline $\mathrm{ZnPc}$ & 0 & 2.68 & -60.00 \\
\hline $\mathrm{ZnNc}$ & 45 & 3.35 & -49.94 \\
\hline
\end{tabular}

potential surface is a quite useful tool to evaluate the interaction between compounds ${ }^{32}$ (Figure S2, SI section), $\mathrm{N}$-meso correspond to negatively charged regions, which may allow stronger interaction with the cation $\mathrm{Zn}^{\mathrm{II}}$ from the other monomer. The distance between molecules in crystals is about $3.23 \AA$ for $\mathrm{ZnPc}^{33}$ and $3.29 \AA$ for $\mathrm{ZnNc}^{34}$ Previous computational simulations furnished the same conformation we have obtained for dimer $0^{\circ}$, but dimer inner distance is greater than that we have found. Besides that, interaction energies can vary, depending on the methodology employed ${ }^{32}$ it was found $9.21 \mathrm{~kJ} \mathrm{~mol}^{-1}\left(2.2 \mathrm{kcal} \mathrm{mol}^{-1}\right)$ with B3LYP/6-31G(d) and $32.55 \mathrm{~kJ} \mathrm{~mol}^{-1}\left(7.8 \mathrm{kcal} \mathrm{mol}^{-1}\right)$ with B3LYP/LanL2MB, both without basis set superposition error (BSSE) correction. On the other hand, Gantchev et al. ${ }^{35}$ obtained $155 \mathrm{~kJ} \mathrm{~mol}^{-1}\left(37.1 \mathrm{kcal} \mathrm{mol}^{-1}\right)$ using molecular mechanics. The higher interaction energies obtained here and the smaller distance between monomers may be due to the slightly larger basis set and, mainly, to the inclusion of dispersion corrections. These corrections are quite important since the energy obtained for larger distances of interaction (dimer $45^{\circ}$ ) is closer to the results derived from molecular mechanics simulations.

In brief, here we have found conformations that correspond to lower $\left(\right.$ dimer $0^{\circ}$ ) and higher (dimer $45^{\circ}$ ) bound estimate in relation to the dimer inner distance. Further studies, mainly using calorimetric experiments and molecular dynamics simulations, ${ }^{36}$ are needed to propose other dimer conformations and include explicitly solvent molecules, to better evaluate energetics and conformations of dimers and higher aggregates. However, these bound estimates are already useful to evaluate tendencies of the effect of interacting molecules on absorption spectra.

TD-DFT spectra for those conformations are presented in Figure 10 along with experimental spectrum for $8.4 \times 10^{-7} \mathrm{~mol} \mathrm{~L}^{-1} \mathrm{ZnPc}$ and $\mathrm{ZnNc}$ solutions and with monomer theoretical lines (same adjustments for monomer and dimer theoretical spectra to fit experimental data). As can be seen, dimer main transitions are quite closer to the monomer main transition, almost all together under the experimental Q-band, especially the $0^{\circ}$ dimer, the most stable one. It is worth noting that this dimer Q-band transition is no more degenerated, since it involves molecular orbitals from the two monomers that are not perfectly superimposed (see Table S3 and Figures S4 to S7, SI section).

Other evidence of dimer presence is the smaller bands in experimental spectra (between 600 and $650 \mathrm{~nm}$ for $\mathrm{ZnPc}$ and 650 and $750 \mathrm{~nm}$ for $\mathrm{ZnNc}$ ): theoretical monomer spectra do not present transitions in those regions, only dimers do. The transition closer to Q-band can be attributed to the main transition of dimer $45^{\circ}$. As this is a less stable dimer, its concentration in experimental system may be smaller than the monomer and dimer $0^{\circ}$ concentrations, resulting in this Q-band shoulder. The other band (around $620 \mathrm{~nm}$ for $\mathrm{ZnPc}$ and $680 \mathrm{~nm}$ for $\mathrm{ZnNc}$ ) seems to correspond to the highest energy transitions of dimer $0^{\circ}$. These transitions involve inner molecular orbitals, as HOMO-2 and HOMO-3, especially for $\mathrm{ZnPc}$. These orbitals are located on only one monomer, so the transitions correspond to a charge transfer from one monomer to the whole dimer. The inclusion of 

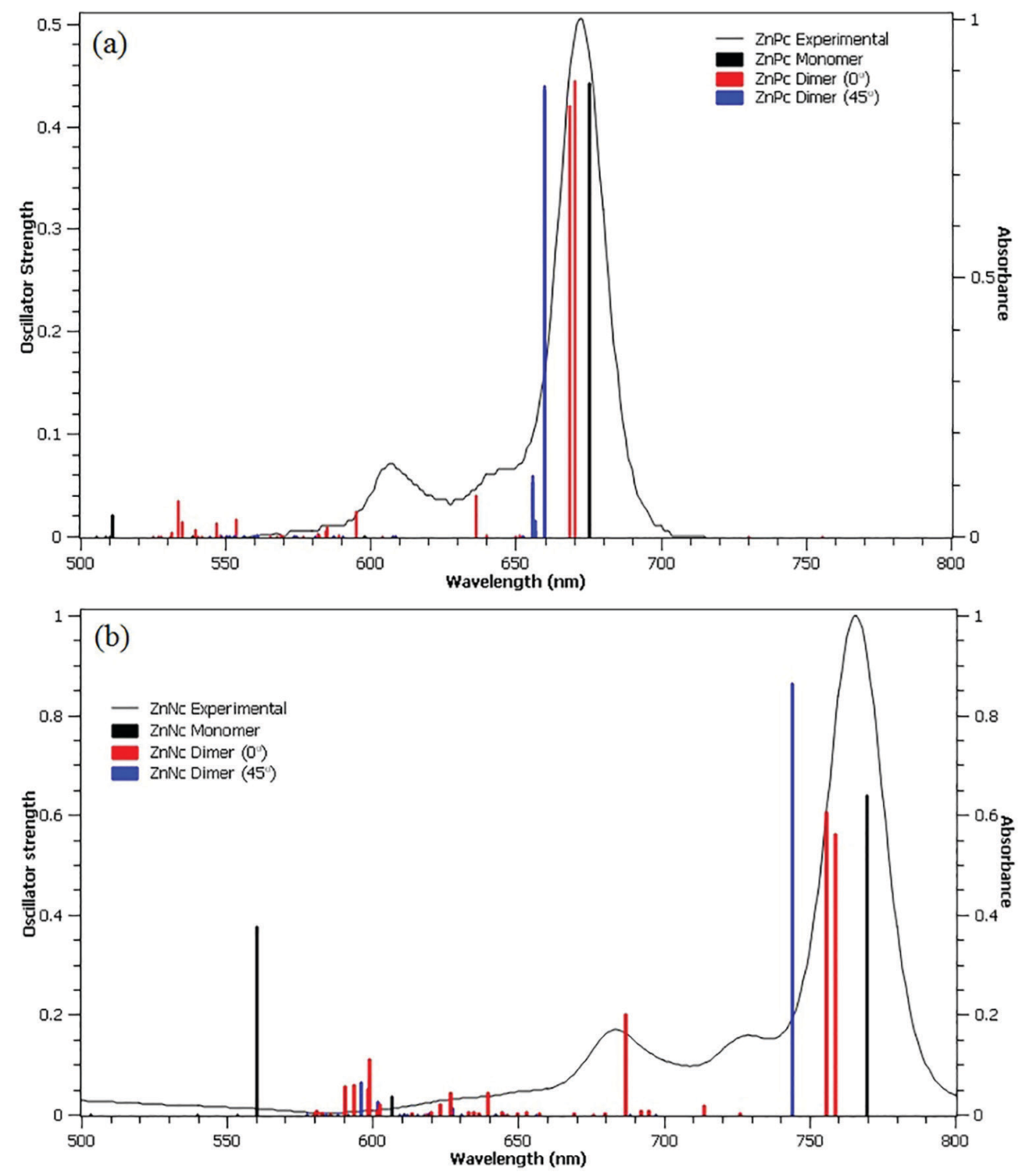

Figure 10. Theoretical absorption spectra calculated with TD-DFT/B3LYP/6-311G(d,p) in DMSO for (a) ZnPc and (b) ZnNc.

thermal effects, as performed by Cabral et al. ${ }^{36}$ for free phthalocyanines, could be conducted to complement the analysis of the influence of aggregation on absorption spectrum.

\section{Conclusions}

In our study we describe the photophysical and photochemical properties phthalocyanine and naphthalocyanines with similar structures to understand the effects of extended $\pi$-conjugation on compounds. Q-band was found to be composed by transitions between HOMO to the degenerated virtual orbitals LUMO and LUMO+1. The extended $\pi$-conjugated system in naphthalocyanine diminishes the energy difference between HOMO and LUMOs and, consequently, causes a bathochromic shift in UV-Vis absorption, emission and excitation bands.
Experimental absorption spectra for all compounds did not show any aggregation behavior in the different solvents employed. Aggregation tendency is observed as the water proportion in DMSO:water solution increment. Naphthalocyanines have more aggregation tendency in DMSO:water solution due to extended $\pi$-conjugated system.

The geometry optimization conducted for $\mathrm{ZnPc}$ and $\mathrm{ZnNc}$ stacking dimers resulted in two conformations. In the first one, monomers are in the same orientation for both compounds $\left(0^{\circ}\right.$ of rotation), but the interaction energy for $\mathrm{ZnNc}$ is quite larger. In the second conformation, monomers are superimposed, but rotated about $45^{\circ}$, and the distance between monomers for $\mathrm{ZnNc}$ is $0.7 \AA$ larger than for $\mathrm{ZnPc}$, which results in a smaller interaction. Further studies involving molecular dynamics simulations are needed to verify other dimer conformations and the relative stability, including substituted compounds. 
Theoretical absorption spectra indicate that main transition of dimers are quite close to the monomer transition, so the dimeric species, even in diluted solutions, are possibly present. Furthermore, other smaller bands present in experimental spectra may correspond to dimer with $45^{\circ}$ of rotation and to higher energy transitions that involve charge transfer from one monomer to the whole dimer.

\section{Supplementary Information}

Data obtained with TD-DFT about the Q-band in electronic absorption spectra and the electrostatic potential surface mapped for each compound are available free of charge at http://jbcs.sbq.org.br as PDF file.

\section{Acknowledgments}

This work was supported by CEM-UFABC, FAPESP (2014/18527-8 and 2012/50680-5) and CNPq (306177/2016-1 and 448125/2014-5).

\section{References}

1. Kim, J.; Soldatova, A. V.; Rodgers, M. A. J.; Kenney, M. E.; Polyhedron 2013, 57, 64.

2. Tau, P.; Ogunsipe, A. O.; Maree, S.; Maree, M. D.; J. Porphyrins Phthalocyanines 2003, 7, 439.

3. Pandey, R.; Kerner, R. A.; Menke, S. M.; Holst, J.; Josyula, K. V. B.; Holmes, R. J.; Org. Electron. 2013, 14, 804.

4. Panicker, N. S.; Smijesh, N.; Philip, R.; Menon, C. S.; Mater. Lett. 2012, 89, 188.

5. Novakova, V.; Reimerova, P.; Svec, J.; Suchan, D.; Miletin, M.; Rhoda, H. M.; Nemykin, V. N.; Zimcik, P.; J. Chem. Soc., Dalton Trans. 2015, 44, 13220.

6. Luan, L.; Ding, L.; Zhang, W.; Shi, J.; Yu, X.; Liu, W.; Bioorg. Med. Chem. Lett. 2013, 23, 3775.

7. Pal, C.; Sharma, A. K.; Cammidge, A. N.; Cook, M. J.; Ray, A. K.; J. Phys. Chem. B 2013, 117, 15033.

8. Lyubimtsev, A.; Iqbal, Z.; Crucius, G.; Syrbu, S.; Taraymovich, E. S.; Ziegler, T.; Hanach, M.; J. Porphyrins Phthalocyanines 2011, 16, 39.

9. Zhang, X.; Xi, Q.; Zhao, J.; J. Mater. Chem. 2010, 20, 6726.

10. Tasso, T. T.; Yamasaki, Y.; Furuyama, T.; Kobayashi, N.; J. Chem. Soc., Dalton Trans. 2014, 43, 5886.

11. Saka, E. T.; Gol, C.; Durmus, M.; Kantekin, H.; Biyiklioglu, Z.; J. Photochem. Photobiol. 2012, 241, 67.

12. Choi, M. T. M.; Li, P. P. S.; Ng, D. K. P.; Tetrahedron 2000, 56, 3881.

13. Suchan, A.; Nackiewicz, J.; Hnatejko, Z.; Waclawek, W.; Lis, S.; Dyes Pigm. 2009, 80, 239.
14. Haris, S. P.; Zhang, Y.; Bourdonnec, B. L.; McCurdy, C. R.; Portoghese, P. S.; J. Med. Chem. 2007, 50, 3392.

15. Pelisson, M. M. M.; Tomé, F. M.; Beltrame, M.; Simioni, A. R.; Tedesco, A. C.; J. Porphyrins Phthalocyanines 2003, 7, 630.

16. Kliesch, H.; Weitemeyer, A.; Müller, S.; Wörle, D.; Liebigs Ann. 1995, 1269.

17. Gurel, E.; Piskin, M.; Altun, S.; Odabas, Z.; Durmus, M.; J. Photochem. Photobiol. 2016, 315, 42.

18. Acar, I.; Biyiklioglu, Z.; Durmus, M.; Katenkin, H.; J. Organomet. Chem. 2012, 708, 65.

19. Staicu, A.; Pascu, A.; Boni, M.; Pascu, M. L.; Enescu, M.; J. Mol. Struct. 2013, 1044, 188.

20. Nas, A.; Dilber, G.; Durmus, M.; Kantekin, H.; Spectrochim. Acta, Part A 2015, 135, 55.

21. Filho, D. A. S.; Coropceanu, V.; Gruhn, N. E.; Neto, P. H. O.; Brédas, J. L.; Chem. Commun. 2013, 49, 6069.

22. Cances, E.; Mennucci, B.; Tomasi, J.; J. Chem. Phys. 1997, $107,3032$.

23. Frisch, M. J.; Trucks, G. W.; Schlegel, H. B.; Scuseria, G. E.; Robb, M. A.; Cheeseman, J. R.; Scalmani, G.; Barone, V.; Mennucci, B.; Petersson, G. A.; Nakatsuji, H.; Caricato, M.; Li, X.; Hratchian, H. P.; Izmaylov, A. F.; Bloino, J.; Zheng, G.; Sonnenberg, J. L.; Hada, M.; Ehara, M.; Toyota, K.; Fukuda, R.; Hasegawa, J.; Ishida, M.; Nakajima, T.; Honda, Y.; Kitao, O.; Nakai, H.; Vreven, T.; Montgomery, J. A., Jr.; Peralta, J. E.; Ogliaro, F.; Bearpark, M.; Heyd, J. J.; Brothers, E.; Kudin, K. N.; Staroverov, V. N.; Kobayashi, R.; Normand, J.; Raghavachari, K.; Rendell, A.; Burant, J. C.; Iyengar, S. S.; Tomasi, J.; Cossi, M.; Rega, N.; Millam, M. J.; Klene, M.; Knox, J. E.; Cross, J. B.; Bakken, V.; Adamo, C.; Jaramillo, J.; Gomperts, R.; Stratmann, R. E.; Yazyev, O.; Austin, A. J.; Cammi, R.; Pomelli, C.; Ochterski, J. W.; Martin, R. L.; Morokuma, K.; Zakrzewski, V. G.; Voth, G. A.; Salvador, P.; Dannenberg, J. J.; Dapprich, S.; Daniels, A. D.; Farkas, Ö.; Foresman, J. B.; Ortiz, J. V.; Cioslowski, J.; Fox, D. J.; Gaussian 09, Revision C.01, Gaussian Inc.: Wallingford, CT, 2009.

24. Grimme, S.; Ehrlich, S.; Goerigk, L.; J. Comput. Chem. 2011, $32,1456$.

25. Grimme, S.; Antony, J.; Ehrlich, S.; Krieg, H.; J. Chem. Phys. 2010, 132, 154104.

26. Neese, F.; Wiley Interdiscip. Rev.: Comput. Mol. Sci. 2012, 2 , 73.

27. Bettanin, F.; Fontinelles, T. A.; Maciel, C. D.; Dias, L. G.; Coutinho Neto, M. D.; Homem-de-Mello, P.; Theor. Chem. Acc. 2015, 134, 152. DOI 10.1007/s00214-015-1732-5.

28. Ueno, L. T.; Jayme, C. C.; Silva, L. R.; Pereira, E. B.; Oliveira, S. M.; Machado, A. E. H.; J. Braz. Chem. Soc. 2012, 12, 2237.

29. Wong, E. W. Y.; Miura, A.; Wright, M. D.; He, Q.; Walsby, C. J.; Shimizu, S.; Kobayashi, N.; Leznoff, D. B.; Chem. - Eur. J. 2012, $18,12404$. 
30. Brena, B.; Puglia, C.; Simone, M.; Coreno, M.; Tarafder, K.; Feyer, V.; Banerjee, R.; Göthelid, E.; Sanyal, B.; Oppeneer, P. M.; Eriksson, O.; J. Chem. Phys. 2011, 134, 074312.

31. Ueno, L. T.; Machado, A. E. H.; Machado, F. B. C.; J. Mol. Struct. 2009, 899, 71.

32. Volpi, R.; Camilo, A. C. S.; Filho, D. A. S.; Navarrete, J. T. L.; Gómez-Lor, B.; Delgado, M. C. R.; Linares, M.; Phys. Chem. 2017, 19, 24202.

33. Lebedeva, N. S.; Mal'kova, E. A.; V'yugin, A. I.; Rev. J. Chem. 2012, 2, 20.
34. Morishige, K.; Araki, K.; J. Chem. Soc., Dalton Trans. 1996, $22,4303$.

35. Gantchev, T. J.; van Lier, J. E.; Hunting, D. J.; Radiat. Phys. Chem. 2005, 72, 367.

36. Cabral, B. J. C.; Cruzeiro, V. W. D.; Coutinho, K.; Canuto, S.; Chem. Phys. Lett. 2014, 595, 97.

Submitted: September 1, 2017 Published online: November 28, 2017 